\title{
VLBI Pulsar Astrometry
}

\author{
R.M. Campbell \\ Joint Institute for VLBI in Europe, Oude Hoogeveensedijk 4, \\ 7991 PD Dwingeloo, the Netherlands
}

\begin{abstract}
I briefly review the means by which VLBI observations can determine the position, proper motion, and parallax of a pulsar, consider a subset of the applications of such results, and highlight recent developments in pulsar gating at JIVE.
\end{abstract}

\section{Introduction}

Interleaved VLBI observations of a pulsar and one or more extragalactic reference sources that lie nearby on the plane of the sky can yield the position of the pulsar with sub-milliarcsecond uncertainties. A series of such observations well spread throughout the seasons can further yield the pulsar's position at a reference epoch $\left(\mathbf{r}_{0}\right)$, its proper motion $(\boldsymbol{\mu})$, and its parallax $(\pi)$ - the latter providing a model-independent distance. Combined with various other independent data, these results can be used to investigate properties of the free-electron distribution in the solar neighborhood and the kinematics of the pulsars themselves. Here, I will review in a very few words how VLBI observations provide an astrometric solution, discuss a small subset of the possible applications, and finish by reviewing results from the initial tests of pulsar gating at the EVN Mk IV data processor at JIVE. See Campbell (2000) and references therein for further discussion of specific applications omitted here for reasons of space and a summary of other astrometric VLBI programs and additional means of determining pulsar distances independently of dispersion measure or Hi absorption.

\section{Estimation of Astrometric Parameters via VLBI}

There are two stages in deriving a full astrometric solution: determining the pulsar's position with respect to known extragalactic reference source(s) at each observing epoch, and calculating $\left(\mathbf{r}_{0}, \boldsymbol{\mu}, \pi\right)$ from the set of these positions at all epochs. We accomplish the first task essentially via modeling the total difference phase-delays between pairs of sources. For the second task, we simultaneously fit for $\left(\mathbf{r}_{0}, \boldsymbol{\mu}, \pi\right)$ in two dimensions using, from each epoch, the Earth's SSBC position taken from a planetary ephemeris and the pulsar positions, uncertainties, and correlations found above.

See Campbell (1995, 2000) and Campbell et al. (1996) for more details on the entire data-reduction procedure and our ongoing program. 


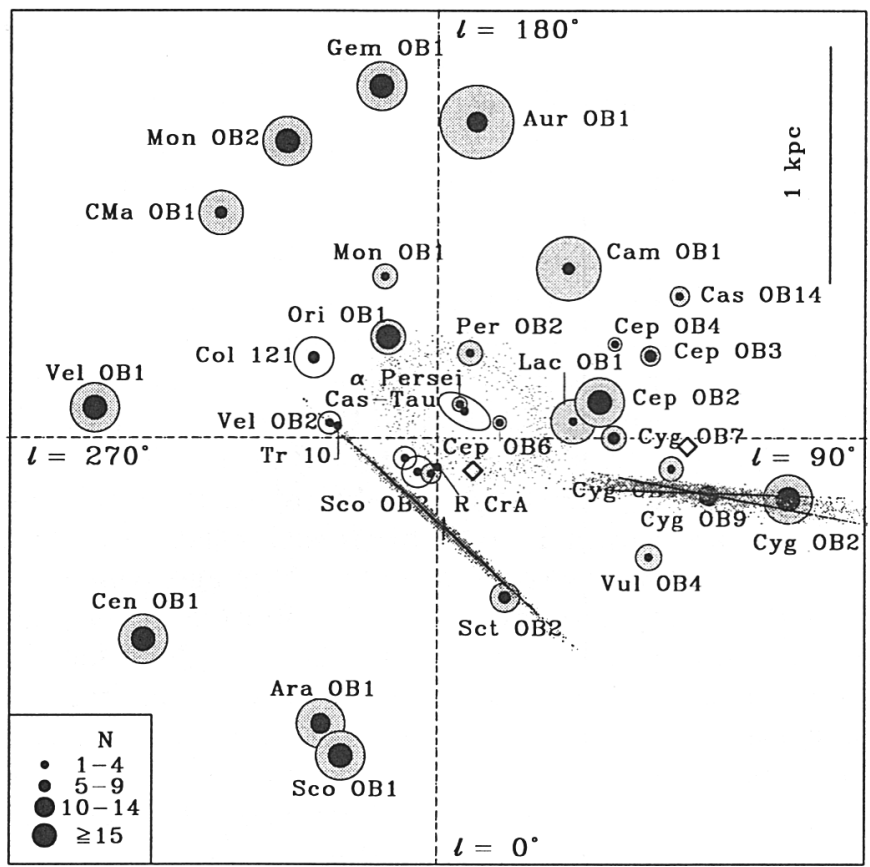

Figure 1. Monte Carlo birthplaces for PSR B1929+10 and PSR B2021+51 in relation to OB associations.

\section{Applications}

The distance, together with the dispersion measure, allows estimation of a lineof-sight averaged electron density, $\left\langle n_{e}\right\rangle$. Let's consider two pulsars in roughly the same direction (lines of sight within $\sim 40^{\circ}$ of each other) but at different VLBIderived distances. PSR B2021 $+51(D=1.06 \mathrm{kpc})$ has $\left\langle n_{e}\right\rangle=0.021 \pm 0.008 \mathrm{~cm}^{-3}$; PSR B1929+10 $(D=0.20 \mathrm{kpc})$ has $\left\langle n_{e}\right\rangle=0.016 \pm 0.005 \mathrm{~cm}^{-3}$. The value for the farther is marginally higher than the outer-disk component of the Taylor \& Cordes (1993) $n_{e}$ model in the vicinity of the Sun $\left(\simeq 0.019 \mathrm{~cm}^{-3}\right)$, and that for the closer marginally less - an inkling of $n_{e}$ structure in the local bubble?

An astrometric solution reduced to the pulsar's local standard of rest (LSR), plus an age estimate, allows tracing the pulsar's motion backwards through $U_{\text {gal }}$ to an inferred birthplace, subject to uncertainties in $\pi, \mu$, and the unmeasured radial velocity $\left(v_{r}\right)$. Figure 1 shows the loci of Monte Carlo birthplaces for PSR B1929+10 and PSR B2021+51 projected onto the Galactic plane, incorporating these three uncertainties. The background is a map of OB associations in the solar neighborhood, taken from de Zeeuw et al. (1999) by permission. The diamonds mark our (current) VLBI positions for the pulsars. The motion of each pulsar was traced backwards in time for the pulsar's spin-down age $\left(\tau_{c}\right)$ : $3.0 \mathrm{Myr}$ for PSR B1929+10 and 2.75 Myr for PSR B2021+51. The two darker linear loci for each pulsar result from considering the sources of uncertainty separately (though plotted, the one due to $\sigma_{\mu}$ is too small to be seen at this scale). The one due to $v_{r}$ lies parallel to the current Sun-pulsar direction, symmetric 
about the mid-point $\left(v_{r}\right.$ taken as $0 \pm v_{t} / \sqrt{2}$, where $v_{t}$ is the transverse velocity in the pulsar's LSR). The grey points represent the Monte Carlo birthplaces considering all three uncertainty sources simultaneously. I plot 3000 Monte Carlo

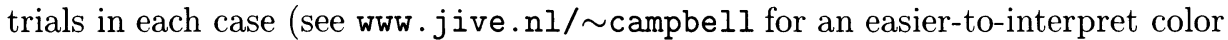
version of this plot). The contribution from $\sigma_{D}$ scales with $D$, but the contribution from $v_{r}$ does not; in the absence of any external information, this will dominate the net birthplace uncertainty for nearby pulsars.

Combining this sort of VLBI pulsar astrometry with Hipparcos astrometry of $\mathrm{OB}$ associations and runaway stars may lead to association with a specific putative progenitor. Hoogerwerf (2000) and Hoogerwerf et al. (2000) have traced PSR B1929+10, the runaway star $\zeta \mathrm{Oph}$, and OB associations backwards in time in a Monte-Carlo fashion. They find subsets of these simulations where the pulsar and the star are within $10 \mathrm{pc}$ of both each other and the center of the Upper Scorpius sub-group of Sco OB2 about $1 \mathrm{Myr}$ ago (this sub-group is the one closest to $\ell=0$ ). Independently, the HI distribution in the sub-group shows an expanding shell-like structure, and $\zeta$ Oph has a high surface He abundance and a large rotational velocity. They show all this is consistent with a binarysupernova scenario for the formation of the pulsar/runaway star: the massive component of a binary explodes as a supernova, and a pulsar goes off with some $v_{\text {kick}}$; the secondary has accreted enriched material and become spun up via Roche-lobe overflow prior to supernova, and moves off as the runaway star with $v \sim v_{\text {orb }}$ once the SN envelope passes reducing $U_{\text {grav }}$ of the erstwhile primary.

\section{Pulsar Gating at the EVN Mk IV Correlator at JIVE}

The goal of pulsar gating on VLBI correlators is to increase the SNR of pulsar observations by integrating only when the pulsar is "on". Sergei Pogrebenko of JIVE has spearheaded the development of pulsar gating capabilities on the JIVE correlator, in collaboration with IRA, Medicina. Pogrebenko \& Tuccari (1994) provide more details about the JIVE gate-fitting concept (for more details about the JIVE correlator itself, see Casse 1999). Very briefly, a Pulsar Gate Generator can provide up to 16 simultaneous gating waveforms, where a waveform is a 1024-long bit-array covering a pulsar's (Earth-centered) period, not merely a start/stop time within the period. Gating circuitry in the individual station units can apply any of the gating waveforms to any of the observed channels (in this context, a specific sub-band/polarization combination). The correlator integrates only for times within each period specified by the gate waveform.

We made initial pulsar-gating test observations of PSR B0329+54, a bright, moderate-period (0.71 s) pulsar, in May 2000, using two dual-polarization Lband subbands. Figure 2 illustrates results from these observations. It plots the correlation functions (single-band delay spectra) for three baselines, integrating $\sim 12$ min of data ( 1000 pulse periods). The left-hand side plots both polarizations (as labeled in the top plot) for the first subband, replicated four times as demarcated by the vertical dotted lines. The replication for each telescope's data occurs in the station unit, and the replications are correlated independently. The right-hand side shows the same data correlated using a gate width $w=P / 2$, where the phase-range of the gate applied to successive replications has a $\mathrm{P} / 4$ overlap as annotated in the top plot. These gates were set such that the pulse 


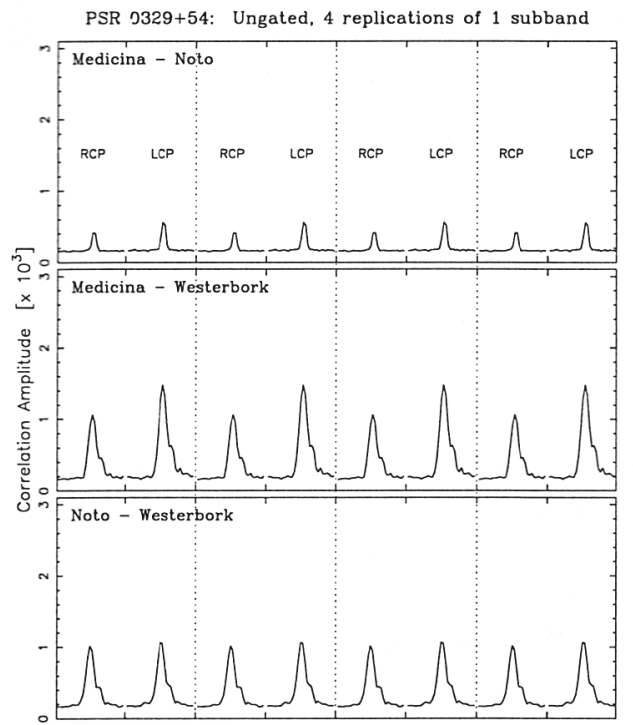

Log [32 per subband/polarization]

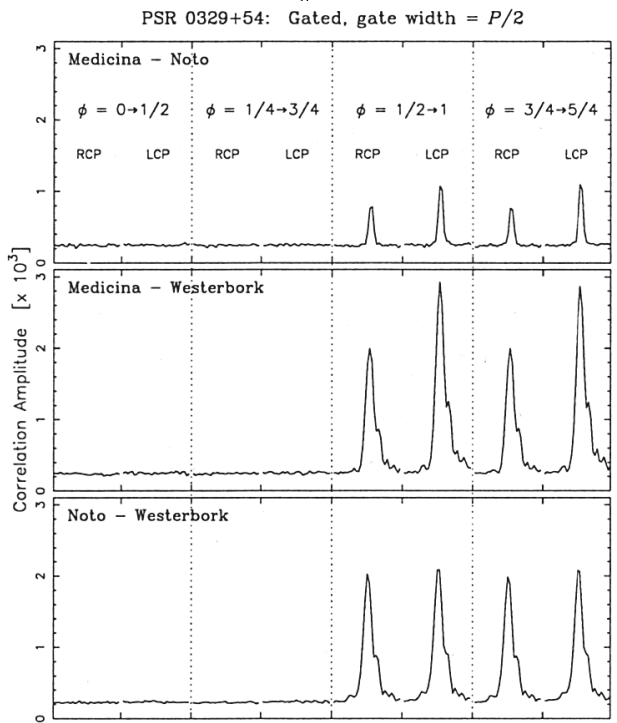

Lag [32 per subband/polarizotion]

Figure 2. Data for PSR B0329+54, before and after gating.

fully lay between 3/4-1. Hence, we get equal response in the third and fourth gates, at an amplitude twice that in the ungated case. Qualitative characteristics of the correlation functions, such as their shapes and relative strengths in $\mathrm{RCP} / \mathrm{LCP}$, are clearly preserved. The reader is reminded that these plots are not integrated pulse profiles: the first two gates have no fringe because we integrate only off-pulse portions of the period. More quantitative tests confirm the expected reciprocal relation between amplitude and $w$ to better than $96 \%$ (the gain in SNR scales only as $w^{-\frac{1}{2}}$, since the noise increases as $\sqrt{w}$ ). Follow-up observations are planned for November 2000 to provide a more stringent test of the phase-model and low-level messaging within the correlator system. Development of pulsar-gating is supported through EC contract ERB FMGE CT98 0101.

\section{References}

Campbell, R. M. 1995, Ph.D. Thesis, Harvard University

Campbell, R. M. 2000, in IAU Colloq. 177, Pulsar Astronomy, ed. M. Kramer, N. Wex \& R. Wielebinski (San Francisco: ASP), 137

Campbell, R. M., Bartel, N., Shapiro, I. I., et al. 1996, ApJ, 461, L95

Casse, J. L. 1999, New Astron.Rev., 43, 503

Hoogerwerf, R. 2000, PhD Thesis, Rijksuniversiteit Leiden.

Hoogerwerf, R., de Bruijne, J. H. J., \& de Zeeuw, P. T. 2000, astro-ph/0007436.

Pogrebenko, S., \& Tuccari, G. 1994, in Proc. Second EVN/JIVE Symposium, ed. A. J. Kus et al. (Torun: TRAO), 119

Taylor, J. H., \& Cordes, J. M. 1993, ApJ, 411, 674

de Zeeuw, P. T., Hoogerwerf, R., de Bruijne, J. H. J., et al. 1999, AJ, 117, 354 